\title{
A B,B,B-Trialkoxyethyllithium Stable towards Fragmentation: a Carboxyl Protected Acetic Acid Dianion Equivalent
}

\author{
Sosale Chandrasekhar* and Chandra Deo Roy \\ Department of Organic Chemistry, Indian Institute of Scieace, \\ Bangalore 560 012, India
}

\begin{abstract}
The novel alkyllithium 1b is not only intriguingly stable towards fragmentation, but also a synthetically useful reagent, complementing current carboxylic ester enolate methodology. Its design is based on interesting mechanistic principles, and barnesses the known stability of the 2,4,10-trioxaadamantane framework.
\end{abstract}

Cyclic acetals and orthoesters are much more stable than their acyclic analogs to hydrolysis. This is thought to be because of a proximity effect, the oxocarbonium ion intermediates in the cyclic cases recyclising very much faster than hydrating. ${ }^{1}$ We report here an application of these mechanistic facts in the design of a novel compound which is also a synthetically useful reagent.<smiles>[R]C12OC(CO)C[C@H](C)C[C@H]1O2</smiles><smiles>C=C1CC2CC(OCl)CC(O1)O2</smiles>

2<smiles>COC(=O)CC(N)c1ccccc1</smiles>

3

1a-h, R: a $\mathrm{H}$, b $\mathrm{CH}_{2} \mathrm{Li}$, c $\mathrm{CH}_{2} \mathrm{Br}$, d $\mathrm{Me}$, e $\mathrm{PhCH}(\mathrm{OH}) \mathrm{CH}_{2}-$, f $\mathrm{PhCOCH}_{2}-$, g PhC(=NOH $) \mathrm{CH}_{2}-$, h $\mathrm{PhCH}\left(\mathrm{NH}_{2}\right) \mathrm{CH}_{2}-$

During mechanistic studies on the origin of the stability of 2,4,10-trioxatricyclo[3.3.1.1,7]decane (1a), ${ }^{2}$ it occurred to us that trioxatricyclodecylmethyllithium $1 \mathrm{1b}$ could be unusually stable to elimination: because of the above proximity effect, the equilibrium between $1 \mathrm{~b}$ and its ring-opened isomer 2 should lie well in favor of $1 b$ which, in fact, is a 'carboxyl-masked $\alpha$-lithio acetic acid'. 
Halogen-metal exchange with $n$-BuLi on bromide $1 \mathrm{c}^{2 \mathrm{~b}}\left(0.2 \mathrm{M}\right.$ in THF $\left./ 0-5{ }^{\circ} \mathrm{C} / \mathrm{N}_{2} / 1 \mathrm{~h}\right)$ did indeed generate $1 \mathrm{~b}$ as shown by its electrophilic reactions. Quenching of $1 \mathrm{~b}$ with water produced methyltrioxatricyclodecane $1 \mathrm{~d}^{2 \mathrm{c}}$, whilst reaction with benzaldehyde produced expected alcohol 1e, both in excellent yields. 1 e could be smoothly oxidised by pyridinium chlorochromate $\left(\mathrm{CH}_{2} \mathrm{Cl}_{2}, 25^{\circ} \mathrm{C}\right)$ to ketone 1f, which could be converted to oxime 1g. Hydride reduction of the oxime produced an excellent yield of $1 \mathrm{~h}$, from which the carboxyl group could be easily retrieved to get methyl B-amino- $B$ phenylpropanoate (3). These transformations are, of course, impossible without the protective trioxatricyclodecyl group, ${ }^{2,3}$ and thus demonstrate the synthetic utility of $1 \mathbf{b}$. Also, $1 \mathbf{b}$ offers an alternative to the use of acetic acid dianion, ${ }^{4}$ but with the advantage of carboxyl protection.

Preliminary studies indicate that $1 \mathrm{~b}$ is rather unreactive towards alkylation, failing to react with MeI, $n-\mathrm{C}_{5} \mathrm{H}_{11} \mathrm{I}$ and $\mathrm{BnBr}\left(\mathrm{THF} / 0-5^{\circ} \mathrm{C} / 2 \mathrm{~h} ; \mathrm{rt} / 18 \mathrm{~h}\right.$ ): a lowering of nucleophilicity by the three electronwithdrawing oxygens, and the neo-pentyl like steric environment, are possible explanations. Further work is planned to extend the scope of the above studies.

\section{EXPERIMENTAL}

Instruments used: Perkin Elmer 781 \& 684, and Hitachi 270-50 (IR); JEOL FX-90Q and Varian T60 (NMR); JEOL MS-DX 303 (GC-MS); Carlo Erba 1160 (elemental analysis). NMR was recorded on $\mathrm{CDCl}_{3}$ solutions in the $0-10 \delta$ range, and acidic protons were often not clearly discemible. IR was recorded for nujol mulls or thin films. Melting and boiling points are uncorrected. Bromide 1c was prepared as reported ${ }^{2 b}$. $n$-BuLi was purchased from Aldrich Chemical Co. Other compounds and reagents were of standard commercial grade. Reaction mixture solutions were dried on $\mathrm{Na}_{2} \mathrm{SO}_{4}$ or $\mathrm{MgSO}_{4}$ unless stated otherwise. ' $\mathrm{H}_{\mathrm{eq}}$ ' and ' $\mathrm{H}_{\mathrm{ex}}$ ' mean equatorial and axial protons respectively.

\section{Lithiation of Bromide 1c.}

1c $(0.117 \mathrm{~g}, 0.50 \mathrm{mmol})$ in dry THF $(2 \mathrm{~mL})$ at $0-5^{\circ} \mathrm{C}$ under dry $\mathrm{N}_{2}$ was treated with $n$-BuLi $(0.5$ $\mathrm{mL}, 1.6 \mathrm{M}$ in hexane, $0.8 \mathrm{mmol}$ ) with stirring which was continued for $1 \mathrm{~h}$. The formation of $1 \mathrm{~b}$ was indicated by reactions of this mixture with electrophiles as detailed below. (1b was prepared on the above scale for these studies).

\section{Reaction with Water.}

Quenching of $1 \mathrm{~b}$ with water $(0.1 \mathrm{~mL})$, concentration in vacuo, extraction with $\mathrm{Et}_{2} \mathrm{O}(3 \mathrm{X} 25 \mathrm{~mL})$, drying and evaporation, and column chromatography of the resulting crude yielded pure $1 \mathrm{~d}(0.056 \mathrm{~g}$, $0.36 \mathrm{mmol}, 72 \%$ ). Mp $125^{\circ} \mathrm{C}$ (from hexane; lit. ${ }^{2 c} 126^{\circ} \mathrm{C}$ ). IR, NMR and HRMS in accord with structure.

\section{Reaction with Benzaldehyde.}

$1 \mathrm{~b}$ was treated with $\mathrm{PhCHO}(0.106 \mathrm{~g}, 1.0 \mathrm{mmol})$ in THF $(1 \mathrm{~mL})$, with stirring which was continued for $3 \mathrm{~h}$ at $0-5^{\circ} \mathrm{C}$ and $14-18 \mathrm{~h}$ at $25^{\circ} \mathrm{C}$. After concentration in vacuo and treatment with $\mathrm{H}_{2} \mathrm{O}(5 \mathrm{~mL})$, the mixture was extracted with $\mathrm{CH}_{2} \mathrm{Cl}_{2}(100 \mathrm{~mL})$, and the extracts dried and distilled. The residue was chromatographed on neutral $\mathrm{Al}_{2} \mathrm{O}_{3}$ (eluent: EtOAc-hexane), and recrystallised (hexane- $\mathrm{CHCl}_{3}$ ), to obtain pure $1 \mathrm{e}(0.105 \mathrm{~g}, 0.40 \mathrm{mmol}, 80 \%)$.

Alcohol 1e. Mp 145-146 ${ }^{\circ} \mathrm{C}$. IR $\left(\mathrm{cm}^{-1}\right)$ 3490. ${ }^{1} \mathrm{H}$ NMR $\delta$ 7.50-7.20 (5 H, m, Ar H), $5.10(1 \mathrm{H}$, dd, $J$ 7.2, $5.4 \mathrm{~Hz}, \mathrm{HO}-\mathrm{CH}$ ), 4.45 (3 H, br. s, C-O-CH), 2.85 (4 H, 'd', $J 14 \mathrm{~Hz}, \mathrm{CH}_{\text {eq }}$ and $\mathrm{OH}, \mathrm{D}_{2} \mathrm{O}$ exchangable), 2.05 ( $\left.2 \mathrm{H}, \mathrm{m}, \mathrm{HO}-\mathrm{C}-\mathrm{CH}_{2}\right), 1.75\left(3 \mathrm{H}, \mathrm{d}, J 14 \mathrm{~Hz}, \mathrm{CH} \mathrm{ux}_{\mathrm{ux}}\right.$ ). MS, $\mathrm{m} / \mathrm{e} 262\left(\mathrm{M}^{+}\right), 245(-$ $\mathrm{OH}), 165,156$. HRMS, $\mathrm{C}_{15} \mathrm{H}_{18} \mathrm{O}_{4}$ requires $m / e$ 262.1200, found 262.1205. Anal. Calcd for $\mathrm{C}_{15} \mathrm{H}_{18} \mathrm{O}_{4}$ : C, 68.68; H, 6.91. Found: C, 69.05; H, 6.94. 


\section{Oxidation of Alcohol 1e.}

1e $(0.524 \mathrm{~g}, 2.0 \mathrm{mmol})$ in dry $\mathrm{CH}_{2} \mathrm{Cl}_{2}$ was stirred with pyridinium chlorochromate $(0.860 \mathrm{~g}, 4.0$ mmol), at $25^{\circ} \mathrm{C}$ for $24 \mathrm{~h}$. The mixture was filtered through $\mathrm{Al}_{2} \mathrm{O}_{3}$ eluting well with $\mathrm{CH}_{2} \mathrm{Cl}_{2}$. Evaporation of the filtrate, and chromatography $\left(\mathrm{Al}_{2} \mathrm{O}_{3}\right)$ of the residue gave pure $1 \mathrm{f}(0.492 \mathrm{~g}, 1.9 \mathrm{mmol}$, 95\%), recrystallised from hexane- $\mathrm{CHCl}_{3}$.

Ketone If. Mp 105-106 ${ }^{\circ} \mathrm{C}$. IR $\left(\mathrm{cm}^{-1}\right)$ 1683. ${ }^{1} \mathrm{H}$ NMR $\delta$ 8.20-8.00 (2 H, m, ArH), 7.60-7.40 (3 H, $\mathrm{m}, \mathrm{ArH}), 4.40$ (3 H, br. s, C-O-CH), $3.30\left(2 \mathrm{H}, \mathrm{s},-\mathrm{CO}-\mathrm{CH}_{2}\right), 2.60\left(3 \mathrm{H}, \mathrm{d}, J 14 \mathrm{~Hz}, \mathrm{CH}_{\mathrm{eq}}\right), 1.68(3$ $\left.\mathrm{H}, \mathrm{d}, J 14 \mathrm{~Hz}, \mathrm{CH}_{\mathrm{ax}}\right)$. MS, $m / e 260\left(\mathrm{M}^{+}\right), 165,147,105$. Anal. Calcd for $\mathrm{C}_{15} \mathrm{H}_{16} \mathrm{O}_{4}: \mathrm{C}, 69.21 ; \mathrm{H}$, 6.19. Found: C, 68.87; H, 6.19.

\section{Preparation of Oxime $1 \mathrm{~g}$.}

A solution of $1 \mathrm{f}(0.130 \mathrm{~g}, 0.50 \mathrm{mmol}), \mathrm{NH}_{2} \mathrm{OH} . \mathrm{HCl}(0.104 \mathrm{~g}, 1.5 \mathrm{mmol})$ and pyridine $(0.1 \mathrm{~mL})$ was refluxed in EtOH ( $2.5 \mathrm{~mL})$ for $1 \mathrm{~h}$, and concentrated in vacuo; the cooled residue was stirred in $\mathrm{H}_{2} \mathrm{O}(5 \mathrm{~mL})$. The resulting crystals were collected, washed with ice-cold $\mathrm{H}_{2} \mathrm{O}(3 \times 15 \mathrm{~mL})$ and recrystallised (hexane- $\left.\mathrm{CHCl}_{3}\right)$, to obtain pure $1 \mathrm{~g}(0.108 \mathrm{~g}, 0.39 \mathrm{mmol}, 78 \%)$.

Oxime 1g. Mp 169-171. IR (cm $\left.{ }^{-1}\right)$ 3232. ${ }^{1} \mathrm{H}$ NMR $\delta$ 7.80-7.20 (5 H, m, ArH), 4.40 (3 H, br. s, $\mathrm{C}-\mathrm{O}-\mathrm{CH}$ ), $3.30\left(2 \mathrm{H}, \mathrm{s}, \mathrm{HON}=\mathrm{CCH}_{2}\right), 2.60\left(3 \mathrm{H}, \mathrm{d}, J 14 \mathrm{~Hz}, \mathrm{CH}_{\mathrm{eq}}\right), 1.68\left(3 \mathrm{H}, \mathrm{d}, J 14 \mathrm{~Hz}, \mathrm{CH}_{\mathrm{ax}}\right)$. MS, $m / e 275\left(\mathrm{M}^{+}\right), 258(-\mathrm{OH})$. HRMS, $\mathrm{C}_{15} \mathrm{H}_{17} \mathrm{NO}_{4}$ requires $m / e$ 275.1158, found 275.1148. Anal. Calcd for $\mathrm{C}_{15} \mathrm{H}_{17} \mathrm{NO}_{4}$ : C, 65.44; H, 6.22; N, 5.09. Found: C, 65.40; H, 6.28; N, 4.74.

Reduction of Oxime $1 \mathrm{~g}$.

$\mathrm{LiAlH}_{4}(0.053 \mathrm{~g}, 1.4 \mathrm{mmol})$ in dry THF $(4 \mathrm{~mL})$ was treated with the oxime $(0.095 \mathrm{~g}, 0.35 \mathrm{mmol})$ in dry THF $(4 \mathrm{~mL})$. The mixture was stirred at $80-85^{\circ} \mathrm{C}$ for $8 \mathrm{~h}$, then concentrated and treated with $\mathrm{H}_{2} \mathrm{O}$. Extraction with $\mathrm{Et}_{2} \mathrm{O}(4 \times 25 \mathrm{~mL})$, drying of the extracts $\left(\mathrm{K}_{2} \mathrm{CO}_{3}\right)$ and evaporation yielded a residue. Chromatography ( $\mathrm{Al}_{2} \mathrm{O}_{3}$ /hexane-EtOAc) gave pure $1 \mathrm{~h}(0.069 \mathrm{~g}, 0.26 \mathrm{mmol}, 74 \%$, viscous liquid).

Amine 1h. IR $\left(\mathrm{cm}^{-1}\right)$ 3358. ${ }^{1} \mathrm{H}$ NMR $\delta$ 7.50-7.00 (5 H, m, ArH), 4.40 (3 H, br. s, C-O-CH), 3.55 (1 $\mathrm{H}, \mathrm{m}, \mathrm{N}-\mathrm{CH}), 2.80\left(2 \mathrm{H}\right.$, br. s, $\left.\mathrm{NH}_{2}\right), 2.50\left(3 \mathrm{H}, \mathrm{d}, \mathrm{J} \cdot 14 \mathrm{~Hz}, \mathrm{CH}_{\text {eq }}\right), 1.95\left(2 \mathrm{H}, \mathrm{m}, \mathrm{N}-\mathrm{C}-\mathrm{CH}_{2}\right), 1.60$ $\left(3 \mathrm{H}, \mathrm{d}, J 14 \mathrm{~Hz}, \mathrm{CH}_{\mathrm{ax}}\right)$. MS, m/e $261\left(\mathrm{M}^{+}\right), 245\left(-\mathrm{NH}_{2}\right), 164,156,106 . \mathrm{HRMS}, \mathrm{C}_{15} \mathrm{H}_{19} \mathrm{NO}_{3} \mathrm{re}-$ quires $m / e$ 261.1365, found 261.1367 .

Preparation of Methyl 3-Amino-3-phenylpropanoate (3).

1h $(0.10 \mathrm{~g}, 0.38 \mathrm{mmol})$ in $5 \mathrm{~N} \mathrm{HCl}(2 \mathrm{~mL})$ was stirred at $25^{\circ} \mathrm{C}$ for $24 \mathrm{~h}$. The mixture was concentrated in vacuo and the resulting residue refluxed in absolute $\mathrm{MeOH}$ for 7-9 h. After neutralisation (solid $\mathrm{NaHCO}_{3}$ ) and concentration in vacuo, $\mathrm{H}_{2} \mathrm{O}$ was added and the resulting mixture ( $\mathrm{pH}$ 8-9) extracted with $\mathrm{CH}_{2} \mathrm{Cl}_{2}(3 \times 25 \mathrm{~mL})$. Drying $\left(\mathrm{K}_{2} \mathrm{CO}_{3}\right)$, evaporation of extracts and chromatography $\left(\mathrm{Al}_{2} \mathrm{O}_{3}\right.$ ) gave pure $3\left(0.05 \mathrm{~g}, 0.28 \mathrm{mmol}, 74 \%\right.$, viscous liquid). ${ }^{5}$

Amino ester 3. IR $\left(\mathrm{cm}^{-1}\right) 3364,1731 .{ }^{1} \mathrm{H}$ NMR $87.40-7.20(5 \mathrm{H}, \mathrm{m}, \mathrm{ArH}), 4.40(1 \mathrm{H}, \mathrm{m}, \mathrm{NCH}), 3.68(3$ $\mathrm{H}, \mathrm{s}, \mathrm{OMe}), 2.64\left(2 \mathrm{H}, \mathrm{d}, \mathrm{J} 7 \mathrm{~Hz}\right.$, accidentally equivalent $\left.-\mathrm{CO}-\mathrm{CH}_{2}\right), 2.00\left(2 \mathrm{H}\right.$, br. s, $\left.\mathrm{NH}_{2}\right) . \mathrm{MS}$, $m / e 179\left(\mathrm{M}^{+}\right)$, 106. HRMS, $\mathrm{C}_{10} \mathrm{H}_{13} \mathrm{NO}_{2}$ requires $m / e$ 179.0946, found 179.0952.

\section{ACKNOWLEDGMENT}

We thank CSIR and DST for generous financial support. 


\section{REFERENCES}

(1) (a) Kirby, A. J.; Martin, R. J. J. Chem. Soc. Perkin Trans. Il.1983, 1627-1632.

(b) Bouab, O.; Lamaty, G.; Moreau, C.; Pomares, O.; Deslongchamps, P.; Ruest, L.

Can. J. Chem. 1980, 58, 567-573.

(2) (a) DeWolfe, R. H. Carboxylic Orthoacid Derivatives; Academic Press: New York, 1970; p 22.

(b) Stetter, H.; Steinacker, K. H. Chem. Ber. 1954, 87, $205-209$.

(c) Stetter, H.; Steinacker, K. H. Chem. Ber. 1953, 86, 790-793.

(3) (a) Bohlmann, F.; Sucrow, W. Chem. Ber. 1964, 97, 1839-1856.

(b) Voss, G.; Gerlach, H. Helv. Chim. Acta. 1983, 66, 2294-2307.

(c) Kunz, H.; Waldmann, H. In Comprehensive Organic Synthesis;

Trost, B. M., Fleming, I., Eds., Pergamon Press: Oxford, 1991; Vol. 6, pp 673-675.

(4) (a) Petragnani, N.; Yonashiro, M. Synthesis 1982, 521-578.

(b) Pfeffer, P. E.; Silbert, L. S.; Chirinko, Jr., J. M. J. Org. Chem. 1972, 37, 451-458.

(5) Pietsch, H. Tetrahedron Lett. 1972, 27, 2789-2792.

(Received in UK 18 February 1994; revised 10 May 1994; accepted 13 May 1994) 